\title{
Retraction Note: Numerical and experimental analyses of repetitive tube ex- pansion and shrinking processed AZ91 magnesium alloy tubes ${ }^{\dagger}$
}

\author{
H. Jafarzadeh* and K. Abrinia \\ Department of Mechanical Engineering, College of Engineering, University of Tehran, Tehran, Iran
}

Retraction note on: Journal of Mechanical Science and Technology 29 (2) (2015) 733 738

DOI 10.1007/s12206-015-0133-y

The Editors of Journal of Mechanical Science and Technology have decided to retract the paper referenced above, because the article contains substantial duplication of text and figures from two previously-retracted papers from the same authors:

RETRACTED: Repetitive tube expansion and shrinking (RTES) as a novel SPD method for fabrication of nanostructured tubes Materials Science and Engineering: A, Vol. 596, 194-199, 2014, H. Jafarzadeh, K. Abrinia, A. Babaei http://www.sciencedirect.com/science/article/pii/S0921509313014111

And

RETRACTED: Applicability of repetitive tube expansion and shrinking (RTES) as a novel SPD method for fabricating UFGed pure copper tubes

Mater. Sci. Eng. A 609, 65-71, 2014, H. Jafarzadeh, K. Abrinia, A. Babaei

http://www.sciencedirect.com/science/article/pii/S0921509314005607

These two articles had themselves been retracted for plagiarism of the following two papers, respectively:

Microstructure and mechanical properties of AZ91D magnesium alloy prepared by compound extrusion

Qiang Chen, Zhixiang Zhao, Dayu Shu, Zude Zhao

Mater. Sci. Eng. A, 528 (2011) 3930-3934

http://www.sciencedirect.com/science/article/pii/S0921509311000360

And

Microstructure, texture and mechanical properties of pure copper processed by ECAP and subsequent cold rolling Sh. Ranjbar Bahadori, K. Dehghani, F. Bakhshandeh

Mater. Sci. Eng. A 583 (2013) 36-42, 10.1016/j.msea.2013.06.061

http://www.sciencedirect.com/science/article/pii/S0921509313007211

On submission of a paper to JMST the authors declare explicitly that their work is original and has not been previously published elsewhere. Published data or figures should be appropriately referenced. The Editors of JMST offer apologies to readers of the journal that this duplication was not detected during the assessment process.

\footnotetext{
$\dagger$ DOI of the original article: DOI 10.1007/s12206-015-0133-y

Corresponding author : H. Jafarzadeh

E-mail address: h.jafarzadeh@ut.ac.ir

(C) KSME \& Springer 2015
} 